\title{
Study of Collagen Birefringence in Different Grades of Oral Squamous Cell Carcinoma Using Picrosirius Red and Polarized Light Microscopy
}

\author{
Pillai Arun Gopinathan,, Ganganna Kokila, ${ }^{2}$ Mahadesh Jyothi, ${ }^{2}$ Chatterjee Ananjan, ${ }^{3}$ \\ Linganna Pradeep, ${ }^{2}$ and Salroo Humaira Nazir ${ }^{2}$ \\ ${ }^{1}$ Oral \& Maxillofacial Pathology, Sri Sankara Dental College, Varkala, Akathumuri, Vennicode, Kerala 695318, India \\ ${ }^{2}$ Oral \& Maxillofacial Pathology, Sri Siddhartha Dental College \& Hospital, Sri Siddhartha Academy of Higher Education, \\ Tumkur 572107, India \\ ${ }^{3}$ Oral \& Maxillofacial Pathology, Vananchal Dental College \& Hospital, Farathiya, Garhwa, Jharkhand 822114, India
}

Correspondence should be addressed to Pillai Arun Gopinathan; arunop7817@gmail.com

Received 1 June 2015; Revised 28 September 2015; Accepted 30 September 2015

Academic Editor: Zhenhe Suo

Copyright (C) 2015 Pillai Arun Gopinathan et al. This is an open access article distributed under the Creative Commons Attribution License, which permits unrestricted use, distribution, and reproduction in any medium, provided the original work is properly cited.

\begin{abstract}
Objectives. The present study was done to evaluate birefringence pattern of collagen fibres in different grades of oral squamous cell carcinoma using Picrosirius red stain and polarization microscopy and to determine if there is a change in collagen fibres between different grades of oral squamous cell carcinoma. Materials and Methods. Picrosirius red stained $5 \mu \mathrm{m}$ thick sections of previously diagnosed different grades of squamous cell carcinoma and normal oral mucosa were studied under polarization microscopy for arrangement as well as birefringence of collagen fibres around tumour islands. Results. It was found that thin collagen fibres increased and thick collagen fibres decreased with dedifferentiation of OSCC $(P<0.0001)$. It was observed that there was change in polarization colours of thick fibres from yellowish orange to greenish yellow with dedifferentiation of OSCC indicating loosely packed fibres $(P<0.0001)$. Conclusion. There was a gradual change of birefringence of collagen from yellowish orange to greenish yellow from well to poorly differentiated squamous cell carcinoma, indicating that there is a change from mature form of collagen to immature form as tumour progresses. Studying collagen fibres with Picrosirius red for stromal changes around tumour islands along with routine staining may help in predicting the prognosis of tumour.
\end{abstract}

\section{Introduction}

Squamous cell carcinoma (SCC) is the commonest type of malignancy affecting oropharyngeal region [1]. SCC is primarily composed of malignant epithelial cells and stroma in which they are dispersed [2]. The birefringence of collagen is related to its physical aggregation which could be altered due to action of collagenases, matrix metalloproteinases (MMPs) secreted by tumour cells [2-4]. In present study, an attempt was made to observe if there is any change in nature of collagen fibres in different grades of oral SCC (OSCC) by determining the ratio of thick to thin fibers as well as their polarizing colours in Picrosirius red stained sections.

\section{Materials and Methods}

Formalin fixed, paraffin embedded tissue blocks of 50 diagnosed cases of OSSC were retrieved from archives of Department of Oral and Maxillofacial Pathology, Sri Siddhartha Dental College, Tumkur. Of these 20 cases were well differentiated SCC (WDSCC), 20 cases were moderately differentiated SCC (MDSCC), and 10 cases were poorly differentiated SCC (PDSCC). 10 cases of clinically uninflamed, normal oral mucosa (NM) were obtained during minor oral surgical procedures. The protocol for the research has been approved by Institutional Ethical Committee.

Two $5 \mu \mathrm{m}$ thick sections were prepared from each paraffin embedded tissue block using semiautomatic microtome. One 


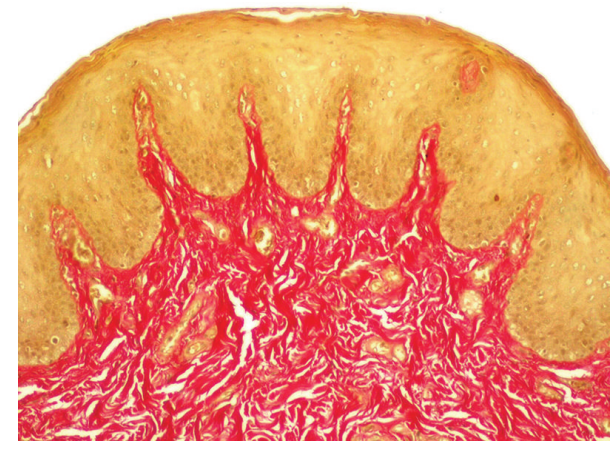

(a)

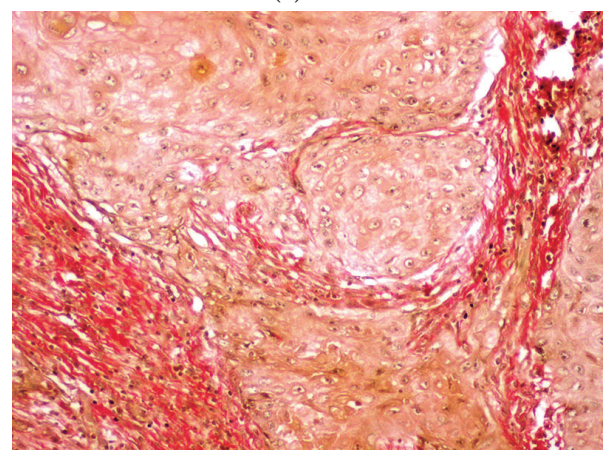

(c)

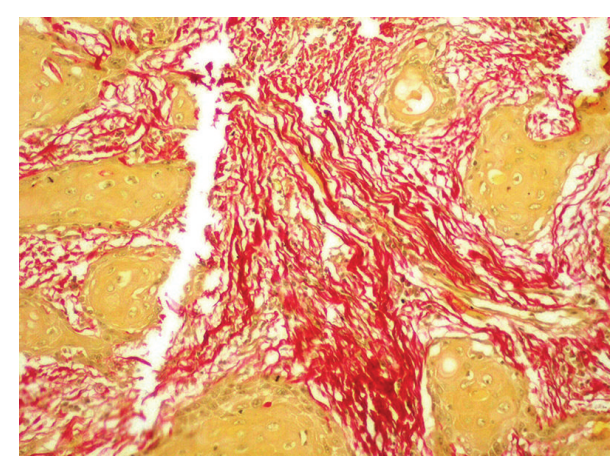

(b)

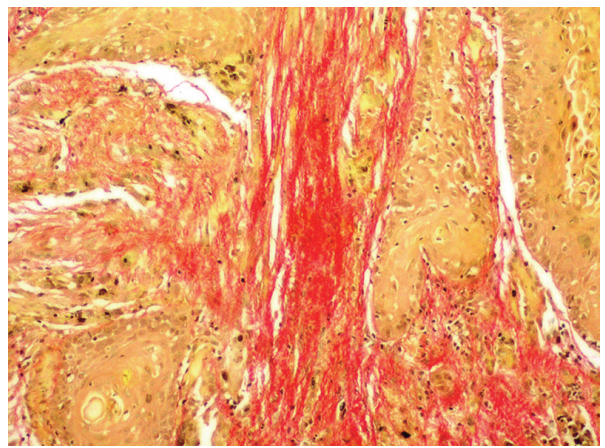

(d)

FIGURE 1: Photomicrograph of PSR stained sections under bright field microscope (10x) showing collagen stained deep red. (a) NM. (b) WDSCC. (c) MDSCC. (d) PDSCC.

of these sections was stained with hematoxylin and eosin and the other with Picrosirius red stain. Hematoxylin and eosin stained sections were viewed under bright field microscopy and the cases were segregated as well, moderately, and poorly differentiated OSCCs according to WHO grading system based on degree of keratinization, cellular and nuclear pleomorphism, and mitotic activity $[5,6]$.

Areas showing epithelial ulceration and dense inflammatory cell infiltration in connective tissue were excluded as inflammation is said to have an impact on packing of collagen fibres. In the normal tissues collagen fibres from lamina propria were studied, while in OSCCS collagen fibres around tumour islands were studied. The polarization colours were determined for 50 thin fibres $(0.8 \mu \mathrm{m}$ or less $)$ and 50 thick fibres $(1.6-2.4 \mu \mathrm{m})$ in each tissue sample. To evaluate change in ratio of thick and thin collagen fibres in different grades of OSCC, randomly 50 fibres were observed and segregated into thick and thin fibres.

2.1. Statistical Analysis. To eliminate subjective bias two observers autonomously evaluated all cases. The obtained scores were tabulated and subjected to statistical analysis using one-way analysis of variance test (ANOVA) for intragroup significance.

\section{Results}

The Picrosirius red stained sections of NM, WDSCC, MDSCC, and PDSCC under bright field microscopy showed collagen fibers stained deep red (Figures 1(a), 1(b), 1(c), and $1(\mathrm{~d})$ ). On examining under polarization microscopy at a lower magnification NM and WDSCC predominantly showed YO birefringence (Figures 2(a) and 2(b)). In MDSCC both YO and GY birefringence was observed (Figure 2(c)), whereas in PDSCC GY birefringence was predominantly seen (Figure 2(d)). At a higher magnification in NM and WDSCC thick collagen fibres with YO birefringence were seen (Figures 3(a) and 3(b)). In MDSCC thick collagen fibres showed YO as well as GY birefringence (Figure 3(c)), whereas in PDSCC thick collagen fibres were predominantly GY (Figure 3(d)).

3.1. Collagen Fibre Arrangement in the Connective Tissue Stroma of NM, WDSCC, MDSCC, and PDSCC. The Picrosirius red stained sections of NM, WDSCC, MDSCC, and PDSCC were studied under polarized light microscopy for type of collagen fibre arrangement in the connective tissue. It was observed that thin fibres increased with dedifferentiation of OSCC which was statistically significant $(P<0.0001)$ (Figure 4$)$. The thick fibres decreased with dedifferentiation of SCC which was also statistically significant $(P<0.0001)$ (Figure 4).

3.2. Polarization Colours of Collagen Fibres in the Connective Tissue Stroma of NM, WDSCC, MDSCC, and PDSCC. Examination of the stroma of NM, WDSCC, MDSCC, and PDSCC in PSR stained sections showed that polarization colours of thin collagen fibres were predominantly greenish yellow 


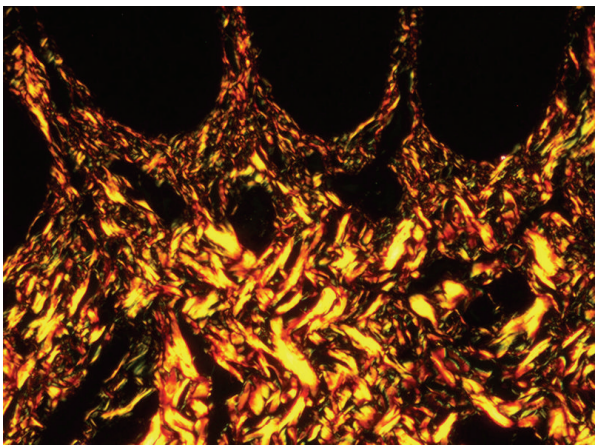

(a)

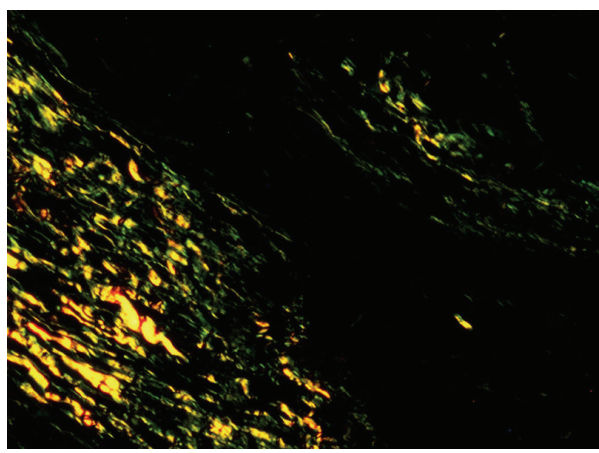

(c)

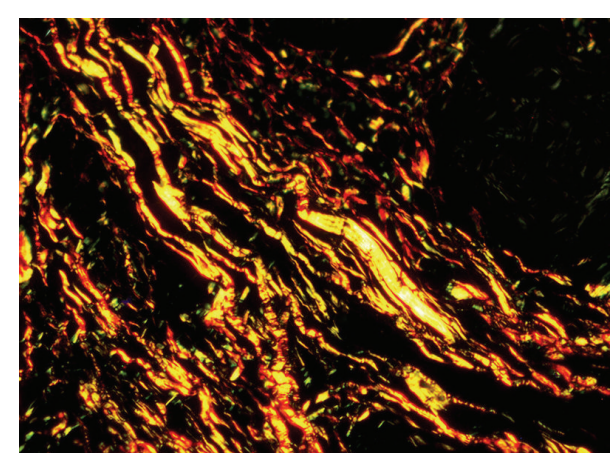

(b)

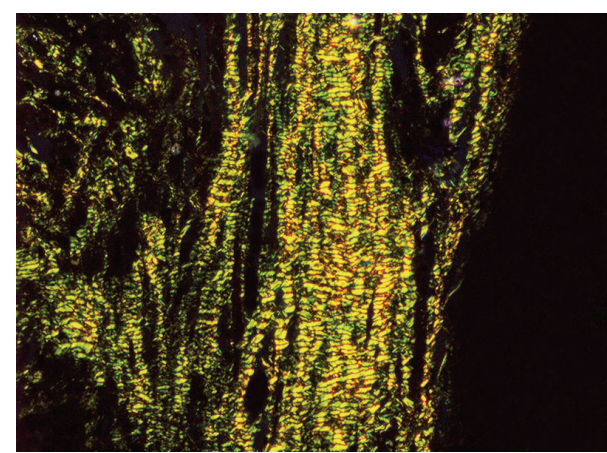

(d)

FIGURE 2: Photomicrograph of PSR stained sections under polarized light microscopy (20x). (a) NM showing YO birefringence. (b) WDSCC showing predominantly YO birefringence. (c) MDSCC showing YO to GY birefringence. (d) PDSCC showing predominantly GY birefringence.

(Figure 5). While polarization colours of thick collagen fibres were mainly yellowish orange in NM and WDSCC, they gradually changed to greenish yellow in MDSCC and PDSCC mainly around tumour islands (Figure 5).

The changes in polarization colours of thin collagen fibres in the stroma of NM, WDSCC, MDSCC, and PDSCC were not statistically significant ( $P$ value: 0.1979 ) (Figure 5$)$. But the changes in polarization colours of thick collagen fibres in the stroma of NM, WDSCC, MDSCC, and PDSCC were statistically significant $(P<0.0001)$ (Figure 5).

\section{Discussion}

OSCC is a common malignancy in India, accounting for $50-70 \%$ of total cancer mortality [7]. Carcinomas are composed of diverse cell populations that are heterogenous for a wide range of characteristics. The tumour progression is accompanied by degradation of the basement membrane and components of matrix which occurs at several stages of metastatic cascade, including local invasion, angiogenesis, and vascular and lymphatic invasion [8].

The mechanical quality of ECM is mainly dependent on its collagenous content and it is the presence of collagen which is considered a main barrier to be cleared away during invasion, thus making room for infiltrating cell mass [9]. MMPs are a group of proteolytic enzymes which degrade most of the components of ECM. The MMP system consists of 23 MMPs which are further divided into five groups, namely, gelatinase, collagenase, stromelysins, membrane type MMPs, and less well characterized MMPs $[8,10]$. The extracellular matrix mainly consists of type I collagen which is about $90 \%$ and type III collagen which is $8-10 \%$ [11]. Electron microscopic studies have shown that type I collagen fibres are coarse and are composed of closely packed thick fibrils, whereas type III collagen forms thin fibres and are composed of loosely disposed thin fibrils [12].

In the present study there were statistically significant $(P<0.0001)$ increase in number of thin collagen fibres and decrease in number of thick collagen fibres which was evident by observing the collagen fibre arrangement in different grades of OSCC. The increase in thin fibres and decrease in thick fibres with dedifferentiation of OSCC could be due to the initial fibroproliferative response and in later stages there will be abnormal collagen production and defective maturation which may promote the neoplastic growth [13]. In extracellular matrix of skin tumour there was an increase in deposition of type I and type III collagen fibres in the stroma of WDSCC, but the destruction of fibrillary structures was more pronounced during the decrease of differentiation from MDSCC to PDSCC [14]. Studies on respiratory neoplasm have also shown that total collagen volume decreases with increasing degree of malignancy from WDSCC, MDSCC to PDSCC and also collagen fibre size decreased in less differentiated SCC [15]. 


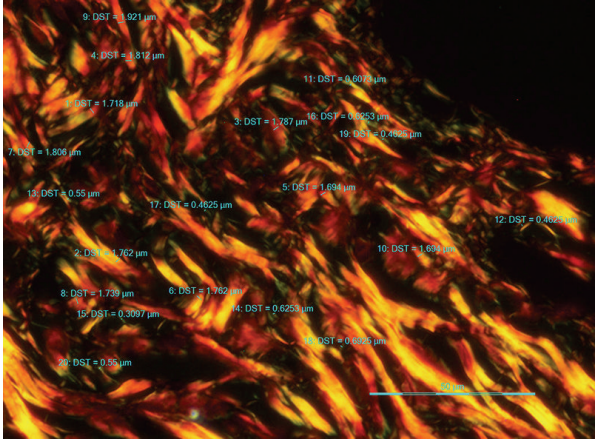

(a)

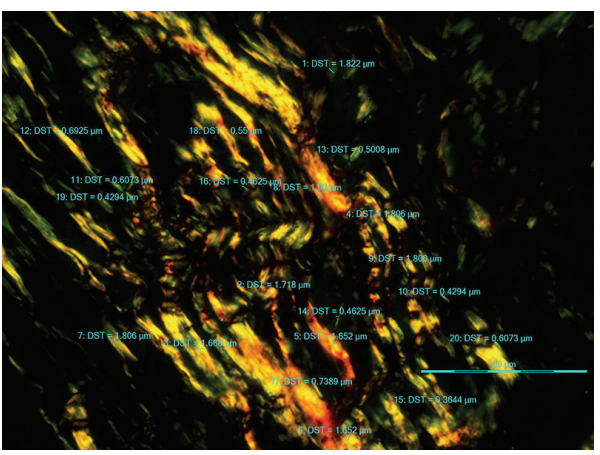

(c)

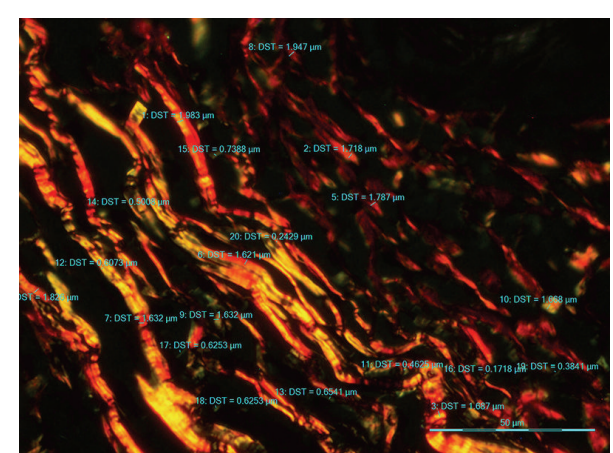

(b)

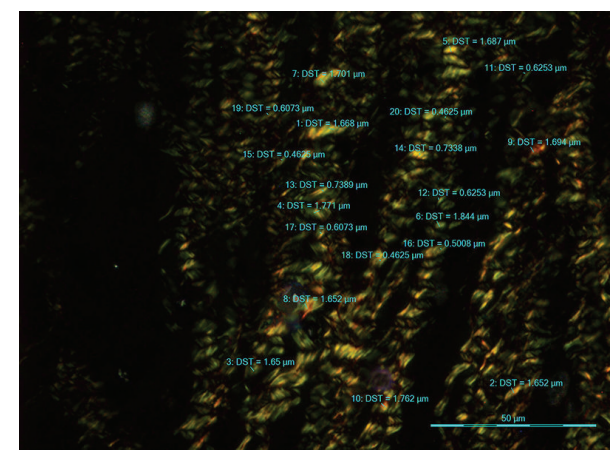

(d)

FIGURE 3: Photomicrograph of PSR stained sections under polarized light microscopy (40x). (a) NM showing predominantly thick YO collagen fibres. (b) WDSCC showing predominantly thick YO and thin GY collagen fibres. (c) MDSCC showing thick YO and thick GY collagen fibres. (d) PDSCC showing thick GY and thin GY collagen fibres.

In a study on maturation of type I and type III collagen fibers in different grades of endometrial adenocarcinoma it was found that in well differentiated adenocarcinomas a distinct layering of type I collagen and bundles of stromal fibres formed a solid homogenous stroma between epithelial cell arrangements. In moderately differentiated adenocarcinomas fibres were irregular and there was a weak deposition of type I collagen adjacent to tumour islets, whereas poorly differentiated adenocarcinomas showed a sparse stroma surrounding individual tumour cells and increased deposition of type III collagen [13].

Lysis of stroma is an essential requirement for invasive growth. It is seen that collagen disintegrates that is they undergo "elastotic degeneration". Electron micrographic studies have shown diffuse collagenolysis and phagocytosis of intact collagen fibrils in the course of carcinoma. Malignant epithelial cells produce various lytic enzymes like cathepsin, elastolytic and glycosaminoglycan degrading enzymes which attack the stroma and induce the fibroblast to synthesize collagenolytic activities [9].

MMPs are a family of proteases and have a generic role in clearing ECM components from the path of a migrating tumour cell. Most of the epithelial tumours express MMPs which are found initially in the surrounding tumour stroma. MMPs exert their effects by proteolyzing the available substrates; for example, MMP-2 induces cell migration, MMPs 2,3 , and 7 release TGF $\beta 1$, and Str-1 (MMP-3) causes cell apoptosis. Other collagenolytic enzymes implicated during tumour growth include lysosomal enzymes particularly acidic cathepsin which attacks collagen fibrils at nonhelical telopeptide regions [16].

A study on collagen in different histological stages of oral submucous fibrosis (OSMF) showed change of polarization colour of thick collagen fibers from YO to GY in advancing connective tissue stages and degrees of epithelial dysplasia [17]. OSMF is a cytokine and growth factor induced disease which increases collagen deposition with advancement of disease [18], while in SCC there is degradation of collagen fibres with advancement of the disease. Thus, it could be hypothesised that there are increase in thin collagen fibres and simultaneous decrease in thick collagen fibres with dedifferentiation of OSCC.

Numerous studies have been done on PSR polarization method and have been used in dental and gingival research to demonstrate pathological changes in collagen [19]. Collagen when stained with PSR and when viewed under polarized light microscopy normally shows thin collagen fibres (type III) which are green to greenish yellow, while thick collagen fibres (type I) range from yellowish orange to orange red polarization colours $[3,4,12]$. The green to greenish yellow colour of both thin and thick fibres suggests that the collagen is loosely packed and orange red colour originates from tightly packed fibres $[3,4]$. The particular colours produced by polarization microscopy of PSR stained section could be 


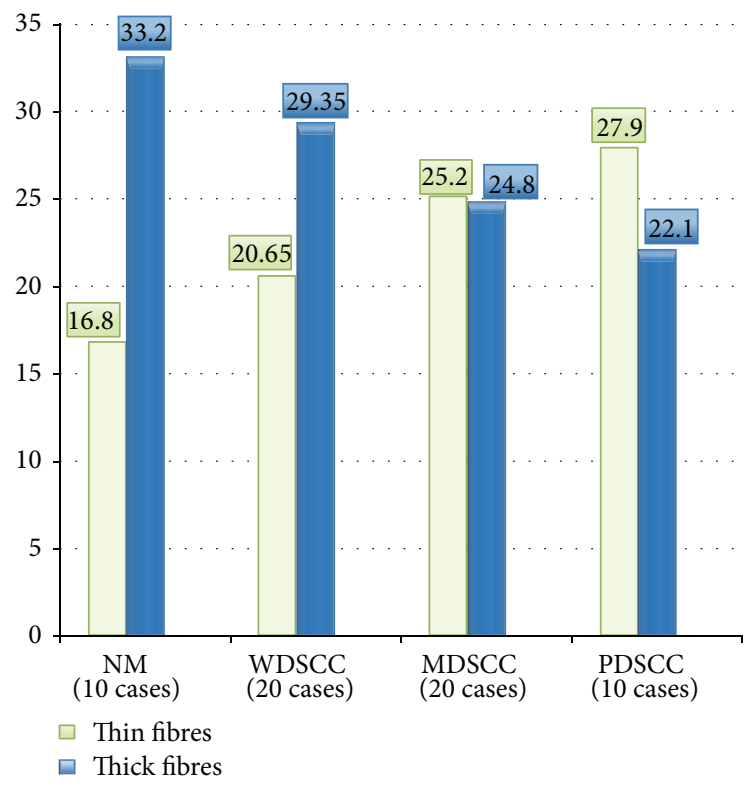

FIGURE 4: Thin and thick fibres arrangement in NM, WDSCC, MDSCC, and PDSCC (out of 50 fibres). ANOVA analysis: $F$ value: 124.31; $P$ value: 0.0000 ; interpretation: highly significant.

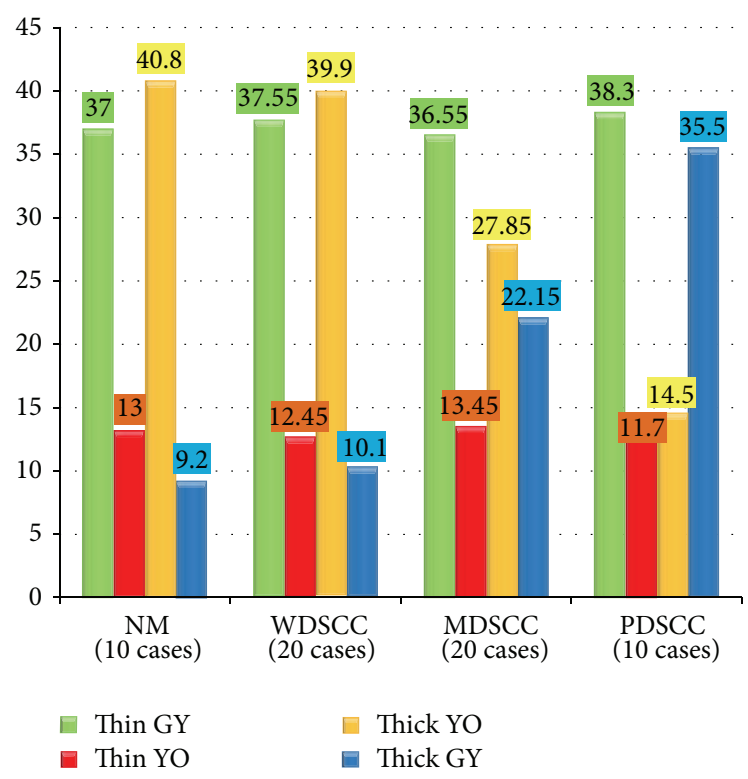

FIGURE 5: Polarization colours of thin and thick fibres in NM, WDSCC, MDSCC, and PDSCC cases. ANOVA analysis: for thin fibres: $F$ value: $1.607 ; P$ value: 0.1979 ; interpretation: not significant. For thick fibres: $F$ value: 224.90 ; $P$ value: 0.0000 ; interpretation: highly significant.

due to fibre size, alignment and packing, cross-linking of fibres, interstitial ground substance, and water content. It is also seen that, in tightly packed and better aligned collagen molecules, a shift to the longer wavelength of polarization colours was seen $[20,21]$.
Polarization colours of collagen fibres in the fibrotic process have shown that, during maturation of fibres, the proteoglycan content changes and dehydration occurs which increases the number of cross-links and stainable side groups; thus the diameter of collagen fibres grows markedly. Finally all these factors enhance the intensity of birefringence and at the same time change their polarization colours. Thus young, very fine type I collagen fibres with weak birefringence appear green in colour similar to the mature type III fibres. They become orange or red in the further maturative stage [22].

Collagen is birefringent, which is used to assess collagen organization and microstructure. The degradation of collagen in pathologies results in disorganization and loss of polarization sensitivity $[23,24]$. Optical technologies like polarized microscopy and Polarization-Sensitive Optical Coherence Tomography are used to assess tissue birefringence which is indicative of disease progression [24]. Polarized microscopy works by passing a natural light through a polarizer called as Nicol prism [25], while Polarization-Sensitive Optical Coherence Tomography works by assessing the polarization state of back-reflected light [24]. Further 2H Double Quantum Filtered (DQF) Nuclear Magnetic Resonance (NMR) spectrum studies have shown that green to greenish yellow colour of thin and poorly packed collagen fibres correlates the narrow component of $2 \mathrm{H} \mathrm{DQF} \mathrm{NMR} \mathrm{spectrum,} \mathrm{while}$ yellow orange red colour pattern of thick well packed collagen fibres corelates the broad component of spectrum [26]. In the present study with respect to relationship between collagenous components in the stroma adjacent to tumour cells, observable changes have been noticed in different histological grades of OSCC. The polarization colours of thin fibres were similar in all the study groups presenting mainly greenish yellow colours. The changes in polarization colours of thin fibres in the stroma of the different study groups were not significant ( $P$ value: 0.1979 ). The polarization colours of thick fibres showed a gradual change from predominantly yellowish orange (YO) to greenish yellow (GY) with dedifferentiation of OSCC. The changes in the polarization colours of thick fibres in stroma of different study groups were significant $(P<0.0001)$.

In the present study the polarization colours of thick fibres were YO 79.8\% in WDSCC and 55.7\% in MDSCC mainly around the tumour islands, which could be due to deposition of collagen fibres in the form of thick bands and closely packed fibrils [2]. The change in polarization colours of thick fibres in MDSCC and PDSCC showed a gradual change in birefringence from YO to GY around the tumour islands, which could be due to loosely packed fibres which might be composed of procollagens, intermediate or pathological collagen rather than normally tight packed fibres. The change in the birefringence of thick fibres in the present study could also be due to adjacent tumour cells which secrete enzymes such as collagenases or MMPs, disorganized stroma, and uninhibited proliferation of dedifferentiated tumour cells with secretion of their abnormal matrix [5]. A similar change in the polarization colours of collagen fibre ranging from $\mathrm{YO}$ to GY was observed in the different grades of OSCC carried out by Aparna and Charu [2]. 
The observations on capsular collagen staining of follicular thyroid carcinomas by PSR showed higher frequency of yellow green collagen fibres at the site of invasion and this could be due to decreased packing density or size of fibre. On the other hand, invasion may result in collagen degradation due to either proteolysis induced by tumour or mechanical pressure due to growth of tumour. It was also noted that orange red fibres were predominant in the noninvaded sites of the follicular thyroid carcinoma [21]. In a study on stromal difference in salivary gland tumour pleomorphic adenoma (PA), polymorphous low grade adenocarcinoma (PLGA), and adenoid cystic carcinoma (ACC) it was found that polarization colours of thin fibres in all the lesions were predominantly greenish yellow, whereas polarization colours of thick fibres in PLGA and ACC were equally divided between greenish yellow and yellow orange and predominance of yellowish orange fibres was seen in PA [15]. A study on human osteosarcoma revealed the presence of type III collagen fibres in anaplastic areas, while both types I and III collagen were present in the fibroblastic areas of the tumour [27].

Green to greenish yellow polarization colours of thick fibres were also noted in pathological conditions like central odontogenic fibroma [28], ameloblastic fibroma [29], odontogenic keratocyst [3], advanced cases of OSMF [18], oxodipine induced hyperplastic gingivitis [30], anastomotic site of intestine [31], and connective tissue nevi [32]. This change in the polarization colours of thick fibres from yellow orange to greenish yellow is considered due to loosely packed fibres which might be composed of procollagens, intermediate or pathological collagen rather than normal tight packed fibres [3, 4, 28-32].

In the present study, an obvious stromal change with the dedifferentiation of the neoplasm was clear with PSR. There were a significant increase of thin and decrease of thick collagen fibres with the dedifferentiation of OSCC. When the birefringence of collagen fibres in the OSCC cases was observed, in well differentiated squamous cell carcinoma, there was deposition of collagen in the form of thick bands revealing thick yellowish orange fibres adjacent to the neoplastic epithelial islands. Gradually in moderately and poorly differentiated squamous cell carcinoma there was a change in the polarization colours of thick fibres from yellowish orange to greenish yellow, where the fibres were fibrillar and more disorganized. This definitively indicates the contribution of the stromal constituents in the progression of the neoplasm which could aid in predicting the prognosis of the tumour.

\section{Conclusion}

On the basis of the present study it may be concluded that with dedifferentiation of OSCC there was a change in the polarization colours of thick fibres from YO to GY due to abnormal collagen production, degradation, and defective maturation which could promote neoplastic progression. In this study, change in the ratio of type I and type III collagen distribution along with change in the birefringence of thick collagen fibres specifically to the degree of dedifferentiation of the neoplasm was seen.

\author{
Abbreviations \\ NM: $\quad$ Normal mucosa \\ WDSCC: Well differentiated squamous cell \\ carcinoma \\ MDSCC: Moderately differentiated squamous cell \\ carcinoma \\ PDSCC: Poorly differentiated squamous cell \\ carcinoma \\ YO: Yellowish orange \\ GY: Greenish yellow \\ PSR: $\quad$ Picrosirius red.
}

\section{Conflict of Interests}

The authors declare no conflict of interests regarding the publication of the paper.

\section{Acknowledgments}

Dr. Pillai Arun Gopinathan extends his great thanks to Dr. Charan Gowda BK, Dr. Smitha, Dr. Sandesh, Dr. Indumati, Dr. Aswathy Raj, Dr. Vivek Pillai, Shri Gopinathan Pillai, Smt Thankamany, Dr. LaxmiDevi BL, Dr. Praveen KS, Prassana Parab, Dr. Manoj S Nair, Dr. Tharun Varghese, Dr. Mithun J Shah, Dr. Swetha Kardalkar, and Dr. Swapnil Shankar Gowda for extending their helping hands in providing him with the required materials and also for the overall fruitful encouragement and advice through the research. He would also like to thank his statistician Dr. Mahesh BH, Assistant Professor in Department of Community Medicine, for his immense help in finalizing his research results and statistics.

\section{References}

[1] S. K. El-Mofty and S. Lewis, "Cancer of the oral cavity and oropharynx," in Surgical Pathology of the Head and Neck, L. Barnes, Ed., vol. 3, pp. 295-341, Informa Health Care, New York, NY, USA, 3rd edition, 2009.

[2] V. Aparna and S. Charu, "Evaluation of collagen in different grades of oral squamous cell carcinoma by using the Picrosirius red stain-a histochemical study," Journal of Clinical and Diagnostic Research, vol. 4, no. 6, pp. 3444-3449, 2010, http:// www.jcdr.net/article_fulltext.asp?issn=0973-709x\&year=2010\& month $=$ December\&issue $=6 \& i d=974$.

[3] I. Allon, M. Vered, A. Buchner, and D. Dayan, "Stromal differences in salivary gland tumors of a common histopathogenesis but with different biological behavior: a study with picrosirius red and polarizing microscopy," Acta Histochemica, vol. 108, no. 4, pp. 259-264, 2006.

[4] A. Hirshberg, S. Sherman, A. Buchner, and D. Dayan, "Collagen fibres in the wall of odontogenic keratocysts: a study with picrosirius red and polarizing microscopy," Journal of Oral Pathology and Medicine, vol. 28, no. 9, pp. 410-412, 1999.

[5] J. A. Woolgar, "Histopathological prognosticators in oral and oropharyngeal squamous cell carcinoma," Oral Oncology, vol. 42 , no. 3, pp. 229-239, 2006.

[6] L. Liaw and H. C. Crawford, "Functions of the extracellular matrix and matrix degrading proteases during tumor progression," Brazilian Journal of Medical and Biological Research, vol. 32, no. 7, pp. 805-812, 1999. 
[7] N. P. Doshi, S. A. Shah, K. B. Patel, and M. F. Jhabuawala, "Histological grading of oral cancer: a comparison of different systems and their relation to lymph node metastasis," National Journal of Community Medicine, vol. 2, no. 1, pp. 136-142, 2011.

[8] E. A. Baker, D. J. Leaper, J. P. Hayter, and A. J. Dickenson, "The matrix metalloproteinase system in oral squamous cell carcinoma," British Journal of Oral and Maxillofacial Surgery, vol. 44, no. 6, pp. 482-486, 2006.

[9] A. van den Hooff, "Stromal involvement in malignant growth," Advances in Cancer Research, vol. 50, pp. 159-196, 1988.

[10] B. L. Ziober, M. A. Turner, J. M. Palefsky, M. J. Banda, and R. H. Kramer, "Type I collagen degradation by invasive oral squamous cell carcinoma," Oral Oncology, vol. 36, no. 4, pp. 365-372, 2000.

[11] B. K. B. Berkovitz, G. R. Holland, and B. J. Moxham, "Oral mucosa," in Oral Anatomy, Histology and Embryology, chapter 14, pp. 223-252, Elsevier, Edinburg, UK, 4th edition, 2009.

[12] G. S. Montes and L. C. U. Junqueira, "The use of the picrosiriuspolarization method for the study of the biopathology of collagen," Memórias do Instituto Oswaldo Cruz, vol. 86, supplement 3, pp. 1-11, 1991.

[13] T. Jussila, S. Kauppila, M. Bode et al., "Synthesis and maturation of type I and type III collagens in endometrial adenocarcinoma," European Journal of Obstetrics Gynecology and Reproductive Biology, vol. 115, no. 1, pp. 66-74, 2004.

[14] F. Stenback, M. Makinen, T. Jussila et al. et al., "The extracellular matrix in skin tumour development-a morphological study," Journal of Cutaneous Pathology, vol. 26, no. 7, pp. 327-338, 1999.

[15] J. Laitakari and F. Stenbäck, "Collagen matrix in development and progression of experimentally induced respiratory neoplasms in the hamster," Toxicologic Pathology, vol. 29, no. 5, pp. 514-527, 2001.

[16] L. Liaw and H. C. Crawford, "Functions of the extracellular matrix and matrix degrading proteases during tumor progression," Brazilian Journal of Medical and Biological Research, vol. 32, no. 7, pp. 805-812, 1999.

[17] K. Ganganna, P. Shetty, and S. E. Shroff, "Collagen in histologic stages of oral submucous fibrosis: a polarizing microscopic study," Journal of Oral and Maxillofacial Pathology, vol. 16, no. 2, pp. 162-166, 2012.

[18] D. Neville and B. Allen, "Epithelial pathology," in Oral and Maxillofacial Pathology, chapter 10, pp. 401-403, Elsevier, Philadelphia, Pa, USA, 3rd edition, 2009.

[19] P. Aggarwal and S. Saxena, "Stromal differences in odontogenic cysts of a common histopathogenesis but with different biological behavior: a study with picrosirius red and polarizing microscopy," Indian Journal of Cancer, vol. 48, no. 2, pp. 211-215, 2011.

[20] D. Dayan, Y. Hiss, A. Hirshberg, J. J. Bubis, and M. Wolman, "Are the polarization colours of Picrosirius red stained collagen determined only by the diameter of the fibres?" Histochemistry, vol. 93, no. 1, pp. 27-29, 1989.

[21] R. Koren, E. Yaniv, D. Kristt et al., "Capsular collagen staining of follicular thyroid neoplasms by picrosirius red: role in differential diagnosis," Acta Histochemica, vol. 103, no. 2, pp. 151$157,2001$.

[22] M. Szendroi, G. Vajta, and L. Kovacs, "Polarization colours of collagen fibres: a sign of collagen production activity in fibrotic processes," Acta Morphologica Hungarica, vol. 32, no. 1, pp. 47$55,1984$.
[23] B. Liu, C. Vercollone, and M. E. Brezinski, "Towards improved collagen assessment: polarization-sensitive optical coherence tomography with tailored reference arm polarization," International Journal of Biomedical Imaging, vol. 2012, Article ID 892680, 10 pages, 2012.

[24] C. Rashidifard, C. Vercollone, S. Martin, B. Liu, and M. E. Brezinski, "The application of optical coherence tomography in musculoskeletal disease," Arthritis, vol. 2013, Article ID 563268, 13 pages, 2013.

[25] J. D. Bancroft and M. Gamble, "Light microscopy", in Theory and Practice of Histological Techniques, chapter 3, pp. 33-52, Churchill Livingstone, 6th edition, 2008.

[26] Y. Sharf, T. Knubovets, D. Dayan, A. Hirshberg, S. Akselrod, and G. Navon, "The source of NMR-detected motional anisotropy of water in blood vessel walls," Biophysical Journal, vol. 73, no. 3, pp. 1198-1204, 1997.

[27] L. C. U. Junqueira, M. T. Assis Figueiredo, H. Torloni, and G. S. Montes, "Differential histologic diagnosis of osteoid: a study on human osteosarcoma collagen by the histochemical picrosirius-polarization method," Journal of Pathology, vol. 148, no. 2, pp. 189-196, 1986.

[28] A. Hirshberg, A. Buchner, and D. Dayan, "The central odontogenic fibroma and the hyperplastic dental follicle: study with Picrosirius red and polarizing microscopy," Journal of Oral Pathology and Medicine, vol. 25, no. 3, pp. 125-127, 1996.

[29] A. Nysaka and D. Dayan, "Ameloblastic fibroma in a young cat," Journal of Oral Pathology \& Medicine, vol. 24, no. 5, pp. 233-236, 1995.

[30] D. Dayan, T. Waner, H. Tal, and N. Abraham, "Polarization microscopy of picrosirius red stained collagen from oxodipineinduced hyperplastic gingiva of beagle dogs," International Journal of Experimental Pathology, vol. 74, no. 3, pp. 225-228, 1993.

[31] M. Y. Rabau and D. Dayan, "Polarization microscopy of picrosirius red stained sections: a useful method for qualitative evaluation of intestinal wall collagen," Histology and Histopathology, vol. 9, no. 3, pp. 525-528, 1994.

[32] H. Trau, D. Dayan, A. Hirschberg, Y. Hiss, J. J. Bubis, and M. Wolman, "Connective tissue nevi collagens. Study with picrosirius red and polarizing microscopy," The American Journal of Dermatopathology, vol. 13, no. 4, pp. 374-377, 1991. 


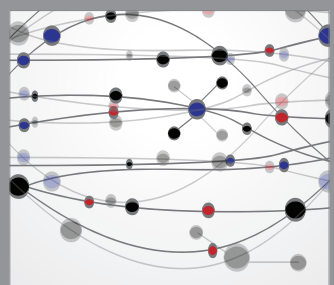

The Scientific World Journal
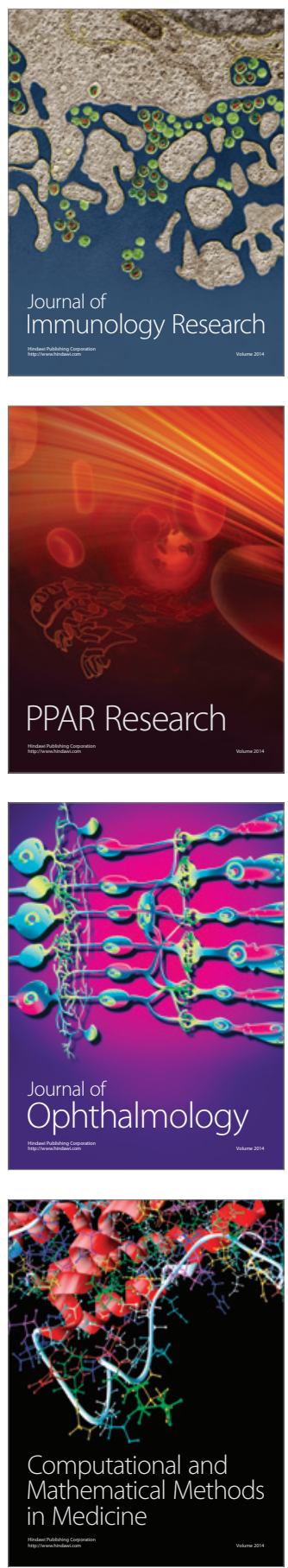

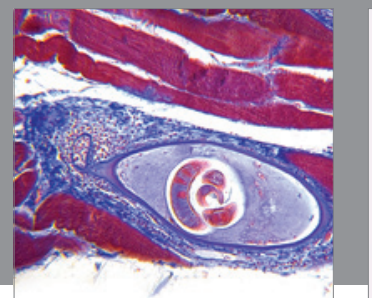

Gastroenterology

Research and Practice
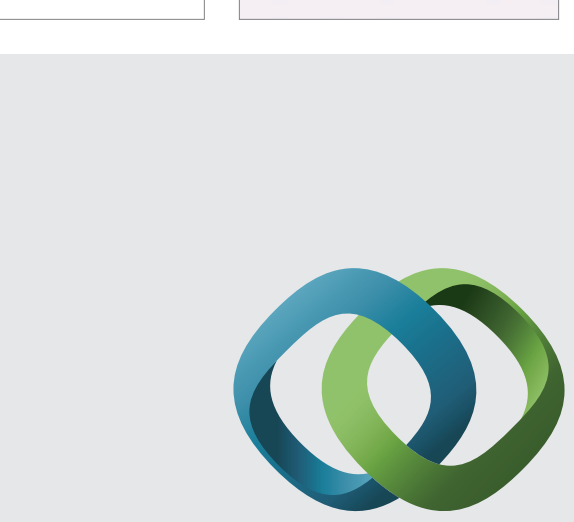

\section{Hindawi}

Submit your manuscripts at

http://www.hindawi.com
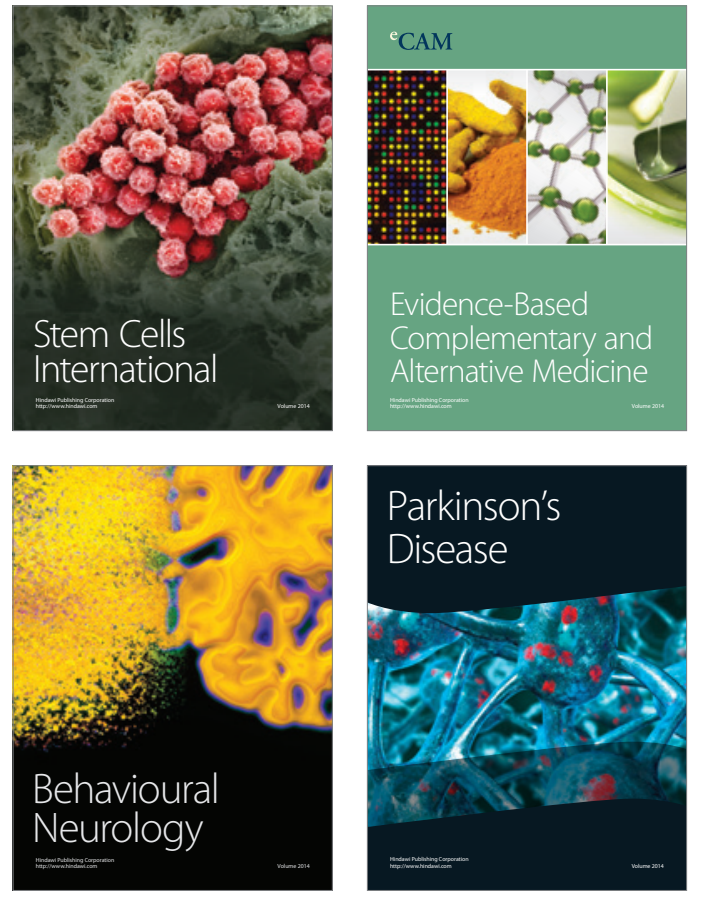
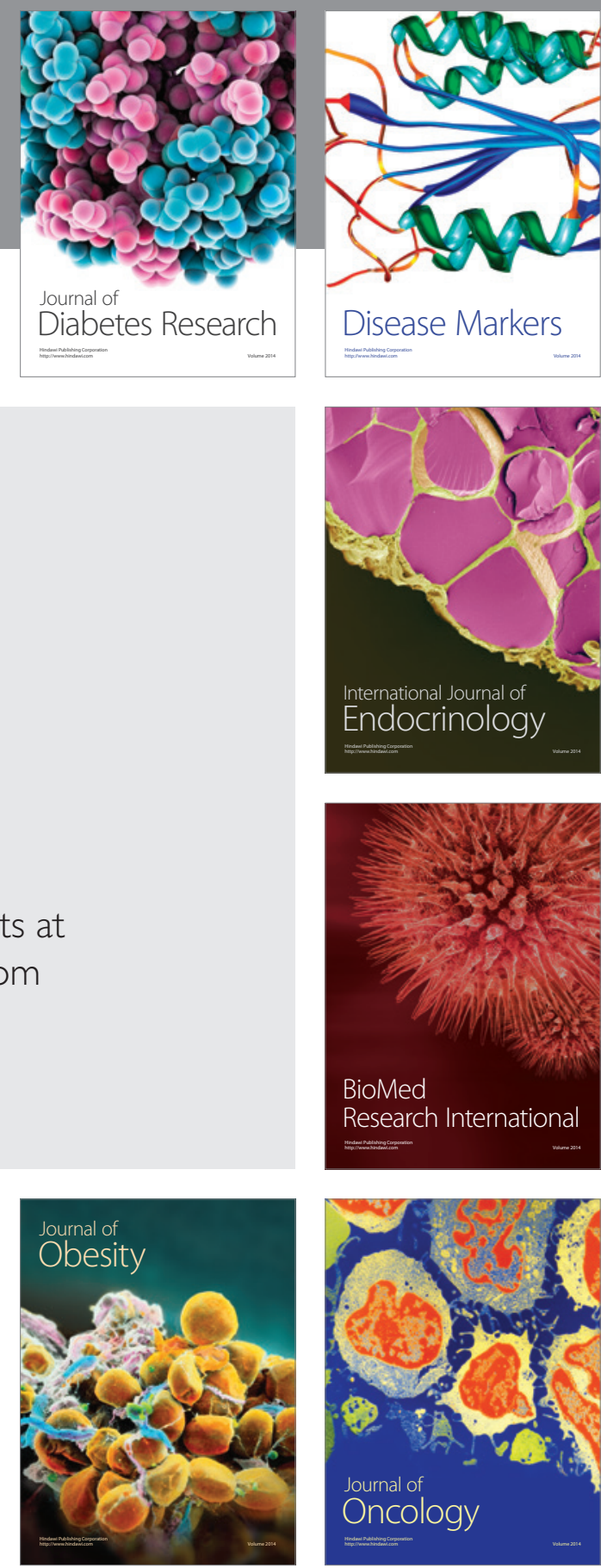

Disease Markers
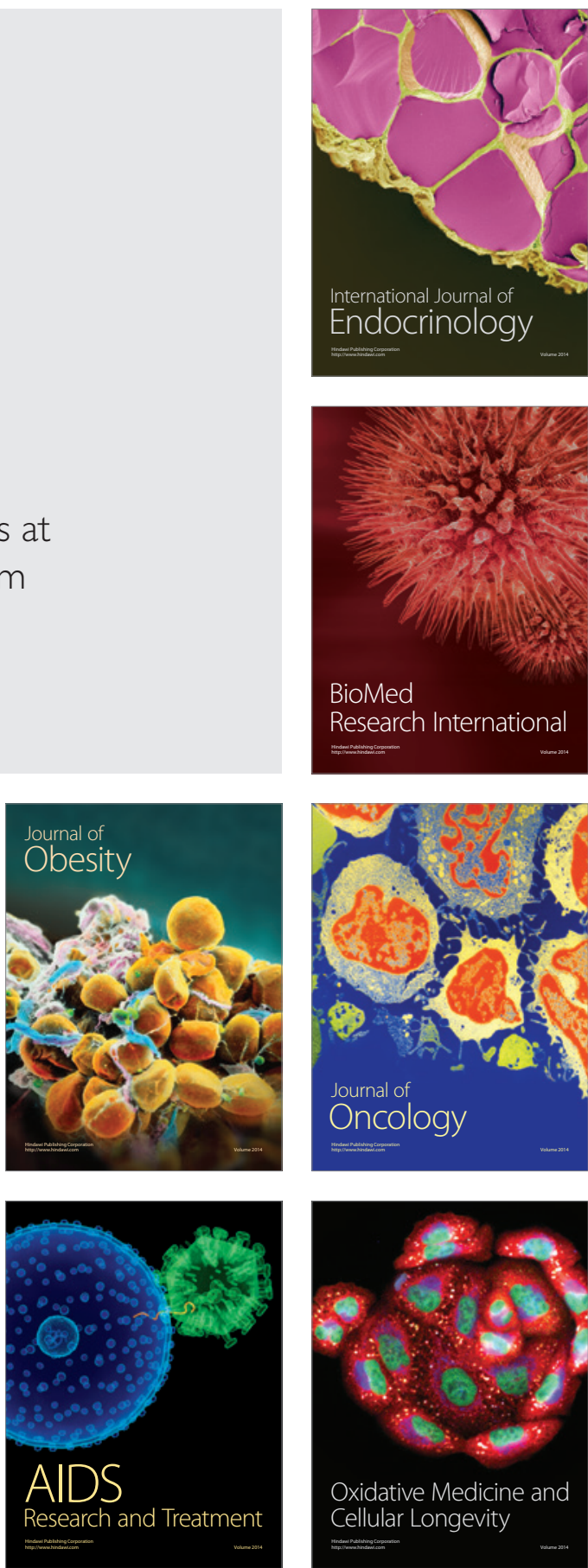